\title{
Staged cardiovascular magnetic resonance for differential diagnosis of Troponin T positive patients with low likelihood for acute coronary syndrome
}

Henning Steen ${ }^{*}$, Media Madadi-Schroeder, Stephanie Lehrke, Dirk Lossnitzer, Evangelos Giannitsis, Hugo A Katus

\begin{abstract}
Background: Cardiac Troponin-T (cTnT) is a cardio-specific indicator of myocardial necrosis due to ischemic or non-ischemic events. Considering the multiple causes of myocardial injury and treatment consequences there is great clinical need to clarify the underlying reason for CTnT release. We sought to implement acute CMR as a noninvasive imaging method for differential diagnosis of elevated cTnT in chest-pain unit (CPU) patients with nonconclusive symptoms and ECG-changes and a low to intermediate probability for coronary artery disease (CAD).

Results: CPU patients $(n=29)$ who had positive CTnT were scanned at 1.5T with a new step-by-step CMR algorithm including cine-, perfusion-, T2-, angiography-and late gadolinium enhancement (LGE) imaging. For comparison patients also underwent echocardiography and coronary angiography if necessary. CMR was conducted successfully in all patients and detected 93\% of cTnT releases of unknown cause, without adverse hemodynamic or arrhythmic events. Acute myocardial infarction was detected in 11, pulmonary embolism in 6, myocarditis in 5, renal disease and cardiomyopathy in 2, storage disorder in 1 patient. In 2 patients CMR was unable to reveal the cause of CTnT elevations. Mean CMR scan-time was $35 \pm 8 \mathrm{~min}$. In 4 patients, CMR led to immediate coronary angiography with correct prediction of the infarct related artery.

Conclusions: We implemented a novel CMR algorithm to show the clinical value and practical feasibility of acute CMR in a non-conclusive patient cohort with unclear CTnT elevation. Since this pilot study has shown the feasibility of CMR in CPU patients, further prospective studies are warranted to compare CMR with other imaging modalities.
\end{abstract}

\section{Background}

Cardiac troponin $\mathrm{T}$ (cTnT) is well established as preferred biomarker for detection of myocardial necrosis due to its absolute cardiospecificity [1]. Numerous clinical trials have established the role of cTnT in patients with suspected acute coronary syndrome for the diagnosis of acute myocardial infarction (AMI) as well as its power for risk strtification of patients with acute coronary syndromes (ACS) with [2] and without ST-segment elevation (STEMI/NSTEMI) [3].

Although the detection of cTnT in blood is specific for myocardial injury, it is not specific as to the cause of

\footnotetext{
* Correspondence: henning.steen@med.uni-heidelberg.de Abteilung Innere Medizin III, Medizinische Klinik, Universitätsklinikum Heidelberg, Heidelberg, Germany
}

(c) 2010 Steen et al; licensee BioMed Central Ltd. This is an Open Access article distributed under the terms of the Creative Commons Attribution License (http://creativecommons.org/licenses/by/2.0), which permits unrestricted use, distribution, and reproduction in any medium, provided the original work is properly cited. coronary cardiac diseases [4] including acute pulmonary embolism [5,6] (PE), acute heart failure [4], myocarditis [7] and toxic injury. If elevated in non-ACS conditions, cTnT elevations are associated with a high cardiac event rate, as shown for example in patients with end-stage renal disease (ESRD) [8].

In all these conditions cTnT elevations are associated with an adverse prognosis with a subsequent substantial need to diagnose and treat the underlying cause of cTnT liberation.

Cardiovascular magnetic resonance (CMR) is a noninvasive comprehensive imaging technique that simultaneously allows assessment of cardiac anatomy, tissue characterization and functional analysis of right and left ventricles (RV, LV). Cardiac dimensions, hypertrophy 
patterns as well as wall motion abnormalities can be easily visualized in breath-hold cine SSFP sequences with superior image quality [9]. Inflammation [10], myocardial hypo-perfusion [11] and infarct-related necrosis [12] are distinctly detectable using T2-edema-or late gadolinium enhancement (LGE) imaging techniques [13]. Furthermore, gadolinium can be utilized for high resolution pulmonary and aortic angiographies to exclude pulmonary embolism [14] or aortic dissection [15].

We sought to implement acute CMR and a novel step-by-step algorithm as a non-invasive diagnostic imaging method for differential diagnosis of elevated cTnT in hemo-dynamically stable patients with non-conclusive symptoms, non-diagnostic ECG, and low to intermediate probability for CAD [16].

\section{Methods \\ Patients}

Patients with an elevated cTnT $(>0.03 \mu \mathrm{g} / \mathrm{L})$ had to fulfil the following criteria for inclusion: 1 . low suspicion of ACS and 2. a) one potential differential diagnosis due to clinical symptoms, or b) certain laboratory findings (c-reactive protein elevation, elevated Wells score [17]). A low likelihood of ACS was defined as a) lack of typical angina, b) low-intermediate probability of CAD $(\geq 2$ cardiovascular risk factors, absent history of CAD) and c) normal/non-diagnostic ECG. Patients were consecutively enrolled in our CPU between January and June 2006. Patients with a high likelihood for ACS were excluded and received standard treatment and diagnostic work-up, such as immediate, early or deferred coronary angiography with or without coronary intervention. AMI was diagnosed using the ESC/AHA/ACC Federation Task Force redefinition of myocardial infarction guidelines [18]. Briefly, AMI required the detection of a rising or falling pattern of cTnT with at least one of the following: symptoms of ischemia, new ST-T changes on ECG, development of $\mathrm{Q}$ waves on ECG, or imaging evidence of new loss of viable myocardium. We excluded asymptomatic patients with ESRD and a constant level of cTnT in two consecutive samples obtained after an interval of at least 6 hours. In ESRD patients, a cTnT-change of $\geq 20 \%$ 6-9 h after presentation has been recommended to indicate an acute condition [19]. cTnT was regarded to be with a recommended diagnostic threshold of $0.03 \mu \mathrm{g} / \mathrm{l}$. The study protocol was in accordance with the Declaration of Helsinki and approved by the local ethical committee. All patients included in the study gave informed written consent.

\section{Study protocol}

After CPU admission and fulfilment of the CMR inclusion criteria, the study patients were consecutively asked and included. We excluded patients with severe dyspnoea, claustrophobia, implanted pacemaker/defibrillators or other metal devices. Patients who were admitted between $7 \mathrm{pm}$ and $8 \mathrm{am}$ were excluded since they were admitted outside the operating CMR hours. Patients were transported to the nearby CMR unit $(\sim 40 \mathrm{~m}$ distance) with a specific MRI trolley-system (Philips PhysioTrak, Best, the Netherlands) that provides the possibility of monitoring blood pressure, heart rate and blood oxygen saturation. The patients' medication, if necessary, was continued during transport and CMR using MR compatible infusion pumps.

During CMR scanning, one technician (B.H., A.W.), one doctor (H.S., S.L., D.L.) and one study assistant (M. M.) were present and analysed the images simultaneously. After diagnosis was made, CMR was stopped and the patient re-transported to the CPU.

Before or after CMR an additional echocardiography exam was accomplished in all patients for verification and comparison with CMR results. Post CMR, suspected ACS or non-coronary differential diagnoses were confirmed by either coronary angiography or 64-slice CT. When after CMR and echo the diagnosis was clear, invasive procedures were not obligatory to minimize patients' X-ray exposure.

\section{The eight-step CMR algorithm}

CMR was performed on a $1.5 \mathrm{~T}$ whole body CMR scanner (Achieva ${ }^{\circ}$, Philips Medical Systems, Best, the Netherlands). The pre-specified algorithm for stepwise evaluation is shown in figure 1 . The algorithm could be followed step-by-step or interrupted at each point to minimize study scan time for the patient.

After scout and reference scans (Step-1), in Step-2 six SSFP-cine images with parallel imaging (slice-thickness $=8 \mathrm{~mm}$; sense-factor $=2$ ) were acquired through the thoracic aorta with subsequent MR angiography if dissection was suspected.

At Step-3, two-chamber and multiple short axis SSFPcine images were scanned for wall motion abnormalities and edema [20] to rule out large AMI. If hypo-or akinesia was present with suspicion of edema, breath-hold T2 black-blood imaging (TSE-SPIR; slice-thickness $=8 \mathrm{~mm}$; Sense-factor $=2.3$ ) and gadolinium three-slice perfusion (Magnevist, Schering Germany;0.05 mmol/kg-bw) imaging (b-FFE; slice thickness $=8 \mathrm{~mm}$;sense-factor $=2$ ) were conducted. Ten minutes after additional 0.15 $\mathrm{mmol} / \mathrm{kg}$-bw gadolinium, multi-slice breath-hold LGE imaging (FFE-T1, TR/TE 2.6/0.9 ms, slice-thickness $=5$ $\mathrm{mm}$, rec. voxel $2 / 2 / 5 \mathrm{~mm}$, sense-factor $=2$, TI-time $=$ 200-300 ms) followed to estimate infarct size and identify the affected coronary territory. CMR results were discussed with the interventional cardiologist for the need of catheterization. 


\section{Scout Scan / Reference Scan}

2. Cine 6 slices thoracic aorta

$$
\longrightarrow \text { Dissection? } \longrightarrow \text { MR Angiography of Aorta }{ }^{* *}
$$

3. Cine 2-Ch / 12 Short Axes

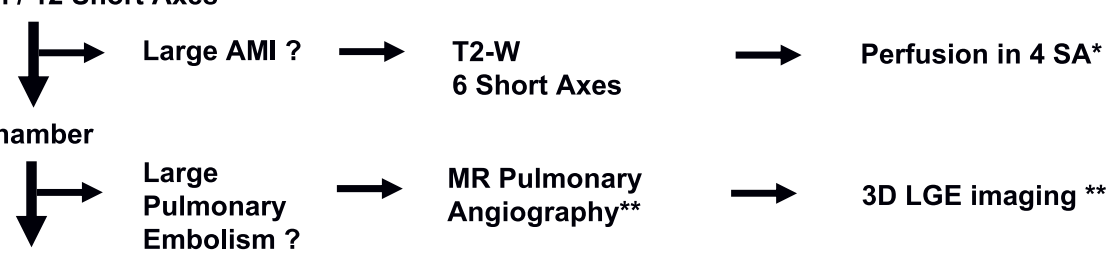

5. T2-W Imaging

Pulmonary
Embolism ? Angiography** $\rightarrow$ 3D LGE imaging *

6 Short Axes

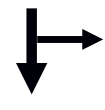

Myo- I

Pericarditis? $\longrightarrow \quad$ MR Perfusion

4 Short axes*

3D LGE imaging **

6. MR Perfusion

4 Short axes*

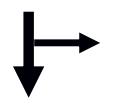

Small AMI ?

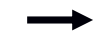

3D LGE imaging **

7. MR Pulmonary

Angiography**

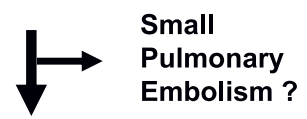

8. Small AMI

Discrete Peri-Myocarditis $\quad \longrightarrow \quad$ 3D LGE imaging **

${ }^{*}=$ Gadolinium DTPA, single dose

${ }^{* *}=$ Gadolinium DTPA, double dose

Figure 1 Eight-step Heidelberg CMR algorithm.

Step-4 contained diagnosis of large PE by performing four-chamber SSFP-cine images to exclude wall motion abnormalities of the RV. If PE was suspected, a non-ECGtriggered 3D-MR pulmonary angiography (3D-FFE, halfscan-data acquisition; rec voxel $=0.7 / 0.7 / 2 \mathrm{~mm}$;sense-factor $=2.2$ ) was accomplished using double dose gadolinium.

After 10 minutes, LGE imaging was conducted to potentially visualize RV myocardial necrosis and pulmonary thrombi.

After triple-rule-out (Step1-4), in Step-5 T2-weighted short axes were imaged to rule-out edema in peri-/myocarditis followed by LGE imaging (see above) to detect patho-gnomonic inflammation patterns.

If no inflammation was seen, Step- 6 contained multislice short axis perfusion imaging to detect small AMI and, in case of a perfusion defect, LGE imaging.
Step-7 looked for small PE performing 3D-MR pulmonary angiography, whereas ten minutes after gadolinium administration Step-8 served as tool for detection of small AMI or peri-/myocarditis. The time points for patient transportation, CMR preparation and scan duration were recorded.

\section{Statistics}

Data were expressed as mean \pm standard deviation or as median with inter-quartile range (IQR; $25-75$ th percentile) if not normally distributed. Differences between two groups were compared by Student's t-test or MannWhitney-U-Test for non-parametrical continuous variables. Continuous variables among more than 2 groups were compared by one-way ANOVA. Categorical variables between groups were compared by chi-square test 
or Fisher's exact test. For all analyses, p-values $<0.05$ were regarded statistically significant. All statistical analyses were carried out using MedCalc 9.4.1.0 (MedCalc Statistical Software bvba, Belgium).

\section{Results}

\section{Study screening and patient characteristics}

Between January and July 2006, 1412 cTnT positive patients were admitted to our CPU. Out of 1,412 eligible patients during this time, 804 were admitted between $7 \mathrm{pm}$ and $8 \mathrm{am}$ (outside the operating times) and could therefore not be included. Of the remaining 608 patients, 110 had a STEMI and received interventional treatment. Of the now remaining 498 patients, 407 patients were clearly diagnosed with NSTEMI with either immediate or delayed invasive diagnostic treatments. Out of the rest of 91 patients, 55 showed either a unequivocal clinical signs of pulmonary embolism, myocarditis or renal failure without cTnT serum level changes cTnT-change of $\geq 20 \% 6-9 \mathrm{~h}$ after presentation. We were thus left with 36 patients who could potentially be included in this pilot study after careful investigations in the CPU. In seven of these 36 patients CMR could not be performed due to claustrophobia or metal implants (4/3 patients). Therefore, 29 patients were included for the final study. The characteristics of the study cohort are shown in table 1 .

\section{Differential Diagnoses detected by CMR}

The CMR protocol was feasible in all 29 cases. During transportation, CMR preparation and scanning, no arrhythmias or hemo-dynamic instability were noted. In our study, the CMR diagnoses were AMI in 11, pulmonary embolism (PE) in 6, peri-/myocarditis in 5, elevated cTnT due to ESRD in 2, cardiomyopathy (CMP) in two and storage disease in 1 patient. In two patients, no diagnosis was found (figure 2). Some clinical CMR examples are given in figure 3.

AMI patients showed significantly more cardiovascular risk factors $(2.9 \pm 0.4, \mathrm{p}=0.04)$, higher admission cTnT $(1.8 \pm 3 \mathrm{ng} / \mathrm{ml})$ and the highest TIMI score $(2.6 \pm 1.3)$ of all groups. CMR detected NSTEMI in nine of eleven cases. In coronary angiography, significant $\mathrm{CAD}$ ( > 50\% luminal obstruction) could be ruled out in one case. Seven cases showed a single-vessel and three showed a two-vessel disease. Nine cases received PCI in at least one lesion.

In six patients PE was detected by CMR. Admission cTnT was significantly lower $(0.15 \pm 0.14 \mathrm{ng} / \mathrm{ml}$; p = 0.023) compared to the AMI group, but had the highest Wells score ${ }^{17}(2.2 \pm 2)$. Since CMR clearly showed PE in four of six patients, only two patients received noninvasive CT for diagnosis confirmation. No heart catheterisation was demanded by the attending physician so that CMR saved invasive procedures in all six cases.
Table 1 Patient demographics and characteristics

\begin{tabular}{|c|c|}
\hline No. of patients & 29 \\
\hline Age (yrs) & $57 \pm 17$ \\
\hline Sex f/m-n (\%) & $9 / 20(31 / 69)$ \\
\hline $\mathrm{BMI}\left(\mathrm{kg} / \mathrm{m}^{2}\right)$ & $25.6 \pm 4.7$ \\
\hline RR syst. (mmHg) & $133 \pm 9$ \\
\hline $\mathrm{RR}$ diast. $(\mathrm{mmHg})$ & $77 \pm 7$ \\
\hline Heart rate (beats/min) & $83 \pm 19$ \\
\hline Fever $>37.5^{\circ} \mathrm{C}$ & $6 / 29$ \\
\hline \multicolumn{2}{|l|}{ Cardiovascular risk factors } \\
\hline Diabetes - n (\%) & $6(21)$ \\
\hline Smoking -n (\%) & $12(42)$ \\
\hline Hypertension - n (\%) & $16(55)$ \\
\hline Hyperlipidemia-n (\%) & $13(45)$ \\
\hline Family history-n (\%) & $13(21)$ \\
\hline TIMI Score & $2.2 \pm 1.2$ \\
\hline Wells Score & $1.0 \pm 1.4$ \\
\hline \multicolumn{2}{|l|}{ Serum parameters } \\
\hline cTnT admission (ng/dl) & $0.9 \pm 1.9$ \\
\hline cTnT max. (ng/dl) & $1.5 \pm 3.9$ \\
\hline Creatinine (mg/dl) & $1.2 \pm 0.6$ \\
\hline GFR (ml/min) & $75 \pm 29$ \\
\hline Leucocytes (/nl) & $10.0 \pm 4.3$ \\
\hline$\ldots$... & $45 \pm 59$ \\
\hline \multicolumn{2}{|l|}{ ECG criteria } \\
\hline Sinus rhythm -n (\%) & $26(90)$ \\
\hline Tachycardia -n (\%) & $7(24)$ \\
\hline Left bundle branch block -n (\%) & $1(3)$ \\
\hline Right bundle branch block -n (\%) & $1(3)$ \\
\hline S1Q3 type $-n(\%)$ & $1(3)$ \\
\hline T-inversion -n (\%) & $13(45)$ \\
\hline ST-deviation -n (\%) & $7(24)$ \\
\hline
\end{tabular}

Five significantly younger patients $(48 \pm 32$ years; $p$ $<0.05)$ with low incidence for cardiovascular risk factors $(1.0 \pm 0.1)$, low Wells $(0.3 \pm 0.7)$ but high serum levels of CRP $(102 \pm 81 \mathrm{mg} / \mathrm{dl})$ revealed peri-/myocarditis on CMR.

Two patients with ESRD (creatine $=2.6 \pm 0.1 \mathrm{mg} / \mathrm{dl}$; MDRD $=31 \pm 0 \mathrm{ml} / \mathrm{min})$, known $\mathrm{CAD}$ and $\mathrm{T}$-wave inversions with ST-deviations, but new onset of atypical chest pain, neither showed wall motion abnormality, edema, perfusion defect nor infarct-typical LGE on CMR. Therefore in both cases no coronary angiography was demanded by the attending physician.

Two elderly female patients without any cardiovascular risk factors, low Wells scores $(0.5 \pm 0.3)$ and moderate cTnT levels $(0.28 \mu \mathrm{g} / \mathrm{l} \pm 0.1)$ revealed either tachyarrhythmia or ST-deviation after psychogenic stress. CMR showed apical ballooning without T2-edema or LGE and classified them as Tako-Tsubo cardiomyopathy.

One younger patient with a moderate cTnT level $(0.31 \mathrm{ng} / \mathrm{ml})$, three CAD risk factors, sinus-tachycardia and $\mathrm{T}$-wave inversions showed a moderately reduced 


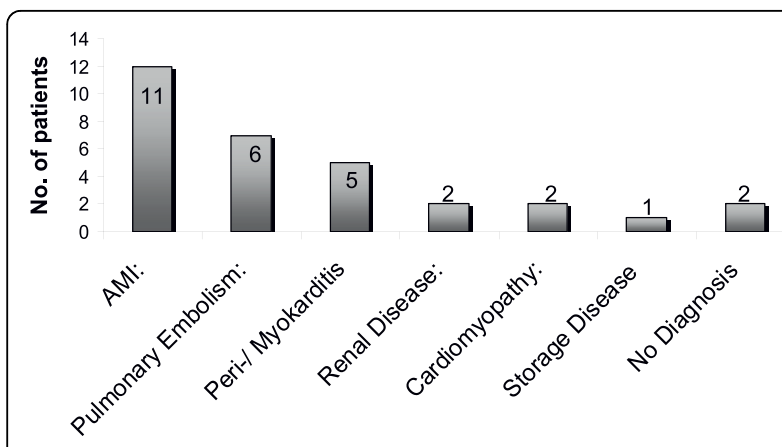

Figure 2 Although clinically in-conclusive the study group comprised 11 AMI-, 6 PE-, 5 peri-/myocarditis-, 2 ESRD and CMP-and one amyloidosis patient. In two patients no diagnosis could be found.

ejection fraction $(\mathrm{EF}=43 \%)$ and symmetric myocardial hypertrophy with thickened heart valves. Surprisingly, LGE imaging showed amyloidosis gadolinium-patterns. The patient refused myocardial biopsy but coronary angiography showed an insignificant LAD lesion. The patient refused any further diagnostics and discharged himself prematurely.

In two elderly patients with low cardiovascular risk profile $(0.5 \pm 0.5)$, marginally elevated $\mathrm{cTnT}(0.03 \mu \mathrm{g} / \mathrm{l})$, good renal function $(1.2 \pm 01 \mathrm{mg} / \mathrm{dl}, 96 \pm 42 \mathrm{ml} / \mathrm{min})$, inconspicuous lab-works, no high grade stenosis on Xray but new arrhythmia with $\mathrm{T}$-wave inversions, the underlying reason for the cTnT elevation could not be identified.

\section{Comparison of diagnostic methods}

In nine of eleven AMI patients, CMR made the correct diagnosis. In one of them cTnT was only minimally elevated $(0.05 \mathrm{ng} / \mathrm{ml})$ with incompliance of breath-hold commands. The other patient showed tachy-arrhythmia with consecutive blurred imaging. Both patients were also misdiagnosed by echocardiography. However, when compared to CMR, echocardiography missed another four AMI patients due to not detected wall motion abnormalities or insufficient acoustic windows. In four cases, patients with CMR diagnosed NSTEMI received an earlier coronary angiography procedure due to large CMR perfusion defects and pronounced LGE patterns. In all AMI cases, CMR predicted the affected coronary vessel correctly. Moreover, CMR distinguished all six patients with $\mathrm{PE}$, whereas echocardiography missed half of them due to insufficient image quality. Since CMR showed the exact localisation of the pulmonary thrombi as well as right ventricular function in at least four of six patients undoubtedly, coronary angiography was not conducted.

Similarly, CMR distinguished conclusively all patients with peri-/myocarditis because of characteristic gadolinium patterns. In only one case with regional wall motion abnormalities and pericardial effusion, echocardiography offered the correct diagnosis. Again, CMR saved coronary angiography procedures in these patients. In two patients, where all three methods could not find a diagnosis, CMR could at least rule out wall motion abnormalities, perfusion defects, edema or significant myocardial necrosis in all 17 AHA segments,

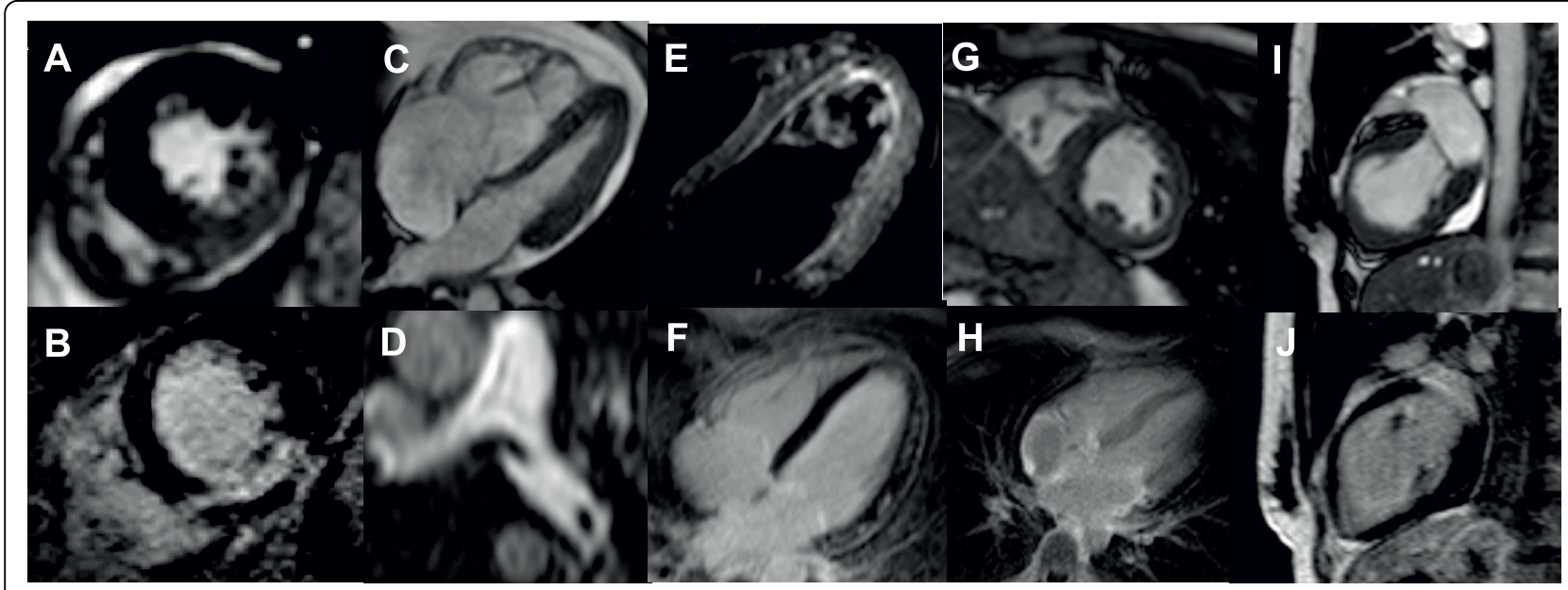

Figure $3 \mathrm{~A}+\mathrm{B}) 63$ year-old patient with inferior wall AMI, T2-edema and hypo-kinesia on short- axis SSFP-images (A) and concomitant LGE (B). C+D) 29 year-old patient with excessively dilated right chambers and systolic dysfunction on SSFP-images (C) revealing thrombi in the proximal left pulmonary artery (D) on pulmonary angiography. On echocardiography the patient showed only insufficient image quality. $\mathbf{E}+\mathbf{F}) 45$ year-old patient with intermitting fever and edema at the LV lateral wall on T2-four chamber images (E) without wall motion abnormalities but clear patchy, infarct-atypical LGE images confirming the diagnosis of myocarditis (F). $\mathbf{G}+\mathbf{H}) 32$ year-old patient with slightly elevated cTnT levels, moderately reduced ejection fraction $(E F=43 \%)$, symmetric myocardial hypertrophy $(\mathbf{G})$ and diffuse LGE patterns $(\mathbf{H})$ suspicious of cardiac amyloidosis. I+J) 62 year-old woman with signs of mid-ventricular ballooning (I) without edema or LGE (J) was classified as tako-tsubo CMP. 
which could not be accomplished by the two other methods. In the two cases of renal dysfunction, CMR again could not show any acute pathology, so that decisions could be made not to proceed with coronary angiography since the coronary status was known from former exams.

\section{CMR time course for differential diagnoses}

In figure 4 seven time bars with average duration of CMR in the different groups are presented. The overall time duration was approximately 1 hour. Mean transportation time to the CMR scanner was $2.5 \pm 1 \mathrm{~min}$, preparation time $15 \pm 2 \mathrm{~min}$, CMR scan time was between $24 \pm 9 \mathrm{~min}$ for PE and $44 \pm 1 \mathrm{~min}$ for patients in which no diagnosis could be found. Transportation back to the CPU after CMR was $11 \pm 3$ min.

\section{Discussion}

For the first time, we show in this prospective pilot study that CMR is useful for confirmation of ACS, adds information on infarct localisation and extent, and may provide a conclusive differential diagnosis in the majority of patients with elevated cTnT concentrations but equivocal signs or symptoms of ACS. Thus, CMR may improve the diagnostic work-up, and may be helpful for patient management. CMR can be completed within 35 minutes, is safe and well tolerated.

\section{CMR for the differential diagnosis of elevated CTnT in patients with low or intermediate probability of ACS}

$\mathrm{CTnT}$ is highly sensitive and characterized by absolute tissue specificity for myocardium. As elevated cTnT is not always due to myocardial infarction, $\mathrm{cTnT}$ in patients with equivocal signs or symptoms for ACS may be caused by differential diagnoses including myocarditis [13], PE [14] or ESRD [19]. Accordingly, confirmation of suspected ACS or accurate differential diagnosis is of paramount importance to avoid unnecessary invasive procedures and to customize therapies. Although the diagnosis of ACS was equivocal in our patients, $30 \%$ had cTnT elevation due to NSTEMI and 9 of 11 cases required subsequent PCI. And although the Wells score demonstrated low clinical pre-test probability for PE, CMR confirmed pulmonary thromboemboli and right ventricular dysfunction in another 6 cases. Therefore, our results demonstrate the usefulness of a comprehensive MR study for differential diagnosis in selected patients with elevated cTnT but with equivocal findings and non-conclusive diagnoses.

CMR identified $93 \%$ of the diagnoses in this CPU patient group. The reason for the minute cTnT rises in two patients $(0.03 / 0.07 \mathrm{ng} / \mathrm{l})$ could not be discovered. In both patients the diagnostic image quality was considerably reduced due to breath-hold incompliance and tachy-arrhythmia. In contrast to previous CT CPU-trials

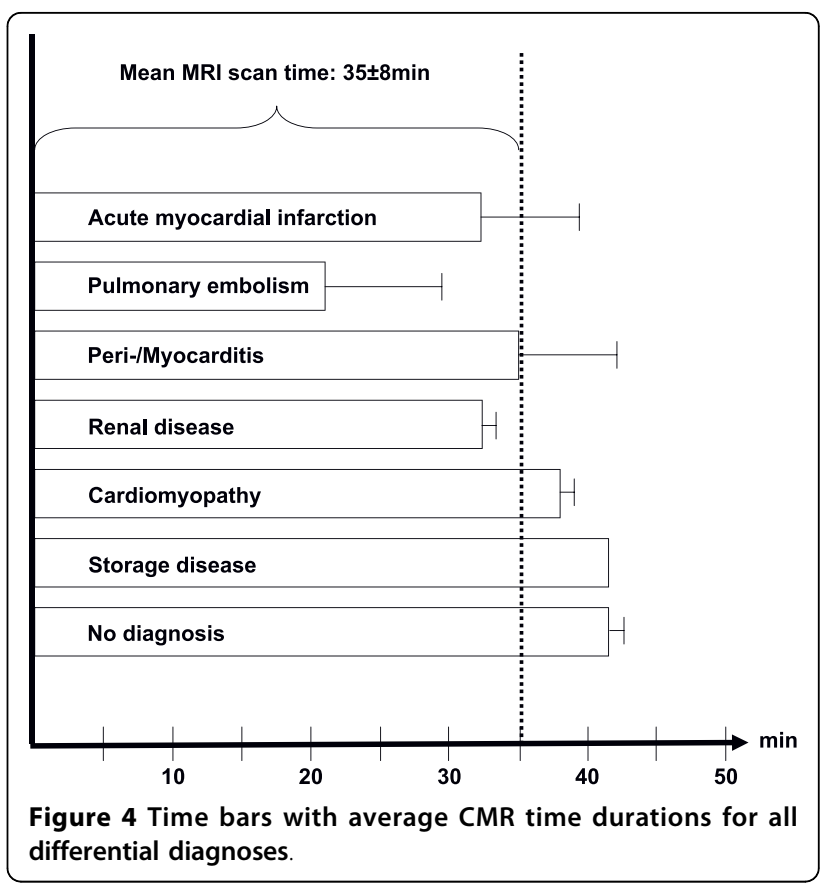

[21], we included also patients with sub-optimal imaging conditions to apply CMR in a clinically realistic setting. Another explanation that we could not find a potential LGE area in the two patients with the slightly elevated cTnT levels could be the employment of our single breath-hold multi-slice-LGE MR sequence holding the disadvantage of a lower image resolution. Therefore, small-sized LGEs could have been overlooked.

Compared to CMR, echocardiography only detected five of eleven AMI patients due to small infarct sizes without wall motion abnormalities. One could argue that perfusion imaging by echocardiography would increase the sensitivity, but even high resolution gadolinium multi-slice perfusion CMR did not reveal noticeable defects. The difference in performance between CMR and echocardiography becomes even more pronounced in patients with PE and peri-/myocarditis, which was the underlying reason in more than $30 \%$ of cTnT releases. If PE did not cause RVdysfunction, echocardiography failed to clarify diagnoses in three patients due to insufficient RV image quality, which is in line with common literature [22]. MR angiography on the other hand enabled visualization of central and peripheral pulmonary thrombi using high-resolution sequences [23]. Also, if peri-myocarditis did not affect wall motion or cause pericardial effusions [22], echocardiography was unable to find the correct diagnosis [24] because of insufficient inflammatory tissue characterisation.

\section{Feasibility of CMR}

All patients were successfully scanned with good image quality and without claustrophobia, with a mean time 
from first scan to diagnosis of $35 \pm 8 \mathrm{~min}$. All CMR scans were conducted between the first and second cTnT measurements (six hours), therefore not causing prolonged patient CPU-stay. CMR could reliably distinguish between hazardous differential diagnoses, which had substantial influence on further diagnostic or therapeutic proceedings. Neither coronary angiography nor echocardiography combined the comprehensive ability of highly reproducible functional as well as and tissue characterisation. Although state-of-the-art CT has superior image resolution for coronary imaging or angiographies, it lacks high-resolution functional and perfusion data so far. Furthermore, inflammatory tissue characterisation cannot be diagnosed appropriately. Thirdly, X-ray and iodine contrast agent administration would have led to a considerable additional radiation exposure in all AMI cases.

\section{Diagnostic decisions and clinical proceedings after CMR}

CMR led to reduced coronary angiography procedures in thirteen cases (6 PE, 5 peri/myocarditis, 2 ESRD), whereas in the 2 ESRD patients the physician decided for non-invasive proceeding because of non-pathological CMR findings. Since CMR predicted all affected coronary AMI territories correctly, it could lead to reduced X-ray exposure in this patient cohort. The concept of CMR implies several essential requirements: 1) short-term clinical access to an CMRsystem with a CPU nearby, 2) safe patient monitoring, 3) CPR trained CMR staff, 4) experienced CMR readers and 5) immediate CMR interpretation. Recently published CPU-CMR studies showed a clear clinical benefit in ACS patients [25]. In conclusion, we implemented the Heidelberg CMR-algorithm to show the clinical value in a clinically inconclusive patient cohort showing unclear cTnT elevations. Since this pilot study has shown the CMR feasibility, further prospective studies comparing CMR with different imaging modalities like $\mathrm{CT}$ are warranted.

\section{Limitations}

We gave gadolinium in two patients with low GFR (34 $\mathrm{ml} / \mathrm{min}$ ), which requires caution because of the potential induction by some contrast agents of nephrogenic systemic fibrosis (NSF) [26]. Coronary angiography was not conducted in all patients for comparison with CMR and echocardiography. After an unequivocal diagnosis finding, patient management was left at the discretion of the attending cardiologist.

\section{List of abbreviations}

CMR: cardiovascular magnetic resonance; CMP: cardiomyopathy; CPU: chest pain unit; CTnT: cardiac Troponin T; Gadolinium: gadolinium-DTPA; LGE: late gadolinium enhancement; LV/RV: left/right ventricle; NSTEMI/STEMI: (Non-) ST-segment-elevation myocardial infarction; PE: pulmonary embolism; CPR: cardiopulmonary resuscitation.
Competing interests

The authors declare that they have no competing interests.

\section{Authors' contributions}

HS coordinated the trial together with MMS. HS analysed the data and was responsible for conception and design of the trial and wrote the manuscript. MMS organised the CMR exams and evaluated the data. SL and DL also scanned the patients and analysed some of the data as well as helped drafting the manuscript. HAK and EG revised the manuscript critically for important intellectual content and finally approved the paper. This paper is not under consideration elsewhere and none of the paper's contents have been previously published. All authors have read and approved the manuscript. All authors have no potential conflict of interests.

\section{Acknowledgements}

We thank Birgit Hoerig and Angela Wochele for high quality cardiac CMRexaminations.

Received: 22 April 2010 Accepted: 14 September 2010

Published: 14 September 2010

\section{References}

1. Katus HA, Remppis A, Neumann FJ, Scheffold T, Diederich KW, Vinar G, Noe A, Matern G, Kuebler W: Diagnostic efficiency of troponine T measurements in acute myocardial infarction. Circulation 1991, 83:902-12.

2. Giannitsis E, Lehrke S, Wiegand UK, Kurowski V, Muller-Bardorff M, Weidtmann B, Richardt G, Katus HA: Risk stratification in patients with inferior acute myocardial infarction treated by percutaneous coronary interventions - the role of admission troponine T. Circulation 2000, 102:2038-44.

3. Hamm CW, Goldmann BU, Heeschen C, Kreymann G, Berger J, Meinertz T: Emergency room triage of patients with acute chest pain by means of rapid testing for cardiac troponine T or troponine I. N Engl J Med 1997, 337:1648-53.

4. Setsuta K, Seino Y, Takahashi N, Ogawa T, Sasaki K, Harada A, Takano T, Kishida $\mathrm{H}$, Hayakawa $\mathrm{H}$ : Clinical significance of elevated levels of cardiac troponin T in patients with chronic heart failure. Am J Cardiol 1999, 84(5):608-11.

5. Perna ER, Macín SM, Parras Jl, Pantich R, Farías EF, Badaracco JR, Jantus E, Medina F, Brizuela M: Cardiac troponin T levels are associated with poor short- and long-term prognosis in patients with acute cardiogenic pulmonary edema. Am Heart J 2002, 143(5):814-20.

6. Giannitsis E, Müller-Bardorff M, Kurowski V, Weidtmann B, Wiegand U, Kampmann M, Katus HA: Independent prognostic value of cardiac troponin $\mathrm{T}$ in patients with confirmed pulmonary embolism. Circulation 2000, 102(2):211-7.

7. Smith SC, Ladenson JH, Mason JW, Jaffe AS: Elevations of cardiac troponin I associated with myocarditis. Experimental and clinical correlates. Circulation 1997, 95(1):163-8.

8. Hamm CW, Giannitsis E, Katus HA: Cardiac troponine elevations in patients without acute coronary syndrome. Circulation 2002, 106:2871-2872

9. Maceira AM, Prasad SK, Khan M, Pennell DJ: Normalized left ventricular systolic and diastolic function by steady-state free precession cardiovascular magnetic resonance. J Cardiovasc Magn Reson 2006, 8:417-426

10. Mahrholdt H, Goedecke C, Wagner A, Meinhardt G, Athanasiadis A, Vogelsberg $H$, Fritz P, Klingel K, Kandolf R, Sechtem U: Cardiovascular magnetic resonance assessment of human myocarditis: a comparison to histology and molecular pathology. Circulation 2004, 109(10):1250-8.

11. Nagel E, Klein C, Paetsch I, Hettwer S, Schnackenburg B, Wegscheider K, Fleck E: Magnetic resonance perfusion measurements for the noninvasive detection of coronary artery disease. Circulation 2003, 108(4):432-7.

12. Kim RJ, Fieno DS, Parrish TB, Chen EL, Simonetti O, Bundy J, Finn JP, Klocke FJ, Judd RM: Relationship of MRI delayed contrast enhancement to irreversible injury, infarct age, and contractile function. Circulation 1999, 100:1992-2002

13. Abdel-Aty $H$, Boyé $P$, Zagrosek $A$, Wassmuth $R$, Kumar A, Messroghli $D$, Bock P, Dietz R, Friedrich MG, Schulz-Menger J: Diagnostic performance of cardiovascular magnetic resonance in patients with suspected acute 
myocarditis: comparison of different approaches. J Am Coll Cardiol 2005, 45(11):1815-22.

14. Fink C, Ley S, Puderbach M, Plathow C, Bock M, Kauczor HU: 3 D pulmonary perfusion MRI and MR angiography of pulmonary embolism in pigs after a single injection of a blood pool MR contrast agent. Eur Radiol 2004, 14(7):1291-6.

15. Nienaber CA, Spielmann RP, von Kodolitsch Y, Siglow V, Piepho A, Jaup T, Nicolas V, Weber P, Triebel HJ, Bleifeld W: Diagnosis of thoracic aortic dissection. Magnetic resonance imaging versus transesophageal echocardiography. Circulation 1992, 85(2):434-47.

16. Braunwald $\mathrm{E}$ : Application of current guidelines to the management of unstable angina and non-ST-elevation myocardial infarction. Circulation 2003, 108(16 Suppl 1):III28-37.

17. Wells PS, Anderson DR, Rodger M, Ginsberg JS, Kearon C, Gent M, Turpie AG, Bormanis J, Weitz J, Chamberlain M, Bowie D, Barnes D, Hirsh J: Derivation of a simple clinical model to categorize patients probability of pulmonary embolism: increasing the models utility with the SimpliRED D-dimer. Thromb Haemost 2000, 83(3):416-20.

18. Braunwald E, Antman EM, Beasley JW, Gibbons RJ, Alpert JS, Faxon DP, Fuster V, Gregoratos G, Hiratzka LF, Jacobs AK, Smith SC Jr: ACC/AHA guideline update for the management of patients with unstable angina and non-ST-segment elevation myocardial infarction-2002: summary article: a report of the American College of Cardiology/American Heart Association Task Force on Practice Guidelines. Circulation 2002, 106:1893-1900.

19. NACB Writing Group, Wu AH, Jaffe AS, Apple FS, Ravkilde J, Tang WH, Christenson RH, Cannon CP, Storrow AB: National Academy of Clinical Biochemistry laboratory medicine practice guidelines: use of cardiac troponin and B-type natriuretic pepatientide or $\mathrm{N}$-terminal proB-type natriuretic pepatientide for etiologies other than acute coronary syndromes and heart failure. Clin Chem 2007, 53(12):2086-96.

20. Kellman P, Aletras AH, Mancini C, McVeigh ER, Arai AE: T2-prepared SSFP improves diagnostic confidence in edema imaging in acute myocardial infarction compared to turbo spin echo. Magn Reson Med 2007, 57(5):891-7.

21. Hoffmann U, Pena AJ, Moselewski F, Ferencik M, Abbara S, Cury RC, Chae CU, Nagurney JT: MDCT in early triage of patients with acute chest pain. AJR Am J Roentgenol 2006, 187(5):1240-7.

22. Goitein O, Matetzky S, Beinart R, Di Segni E, Hod H, Bentancur A, Konen E: Acute myocarditis: noninvasive evaluation with cardiac MRI and transthoracic echocardiography. AJR Am J Roentgenol 2009, 192(1):254-8.

23. Neeb D, Kunz RP, Ley S, Szábo G, Strauss LG, Kauczor HU, Kreitner KF, Schreiber LM: Quantification of pulmonary blood flow (PBF): validation of perfusion MRI and nonlinear contrast agent (CA) dose correction with $\mathrm{H}$ (2)150 positron emission tomography (PET). Magn Reson Med 2009, 62(2):476-87.

24. Goldhaber SZ, Visani L, De Rosa M: Acute pulmonary embolism: clinical outcomes in the International Cooperative Pulmonary Embolism Registry (ICOPER). Lancet 1999, 353(9162):1375-6.

25. Kwong RY, Schussheim AE, Rekhraj S, Aletras AH, Geller N, Davis J, Christian TF, Balaban RS, Arai AE: Detecting acute coronary syndrome in the emergency department with cardiac magnetic resonance imaging. Circulation 2003, 107(4):531-7.

26. Marckmann P, Skov L, Rossen K, Dupont A, Damholt MB, Heaf JG Thomsen HS: Nephrogenic systemic fibrosis: suspected causative role of gadoliniumodiamide used for contrast-enhanced magnetic resonance imaging. J Am Soc Nephrol 2006, 17(9):2359-62.

doi:10.1186/1532-429X-12-51

Cite this article as: Steen et al:: Staged cardiovascular magnetic resonance for differential diagnosis of Troponin T positive patients with low likelihood for acute coronary syndrome. Journal of Cardiovascular Magnetic Resonance 2010 12:51.

\section{Submit your next manuscript to BioMed Central and take full advantage of:}

- Convenient online submission

- Thorough peer review

- No space constraints or color figure charges

- Immediate publication on acceptance

- Inclusion in PubMed, CAS, Scopus and Google Scholar

- Research which is freely available for redistribution

Submit your manuscript at www.biomedcentral.com/submit
Biomed Central 